\title{
mTOR inhibition in breast cancer
}
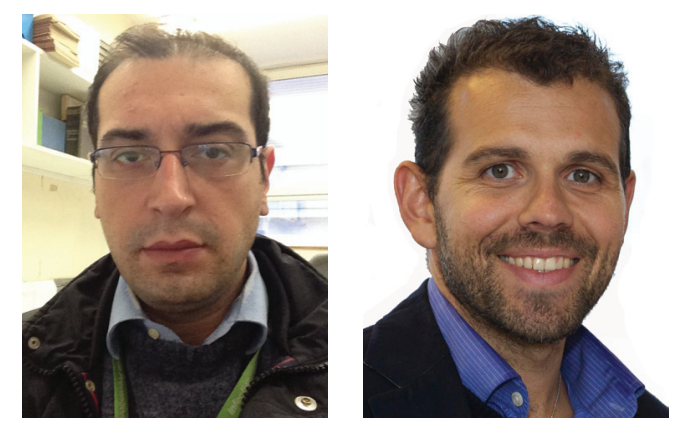

\author{
“The field of PI3K/mTOR \\ inhibition in breast cancer \\ remains highly active with \\ many potentially exciting \\ implications that can \\ revolutionize the clinical \\ management of patients."
}

Panos Papanastasopoulos ${ }^{1}$ \& Georgios Giamas*,1

\begin{abstract}
What is already known
mTOR is a serine/threonine kinase and downstream member of the PI3K/protein kinase B (AKT) and adenosine monophosphate-activated protein kinase pathways, with an essential role in cell growth, survival and autophagy. mTOR is a component of two structurally similar complexes, mTORC1 and mTORC2, which are, however, functionally distinct. mTORC1 promotes mRNA translation and protein synthesis by phosphorylation of ribosomal protein S6 kinase and eIF4E-binding protein 1 and inhibits autophagy. mTORC2 organizes the cellular actin cytoskeleton and regulates AKT phosphorylation, promoting cell survival. AKT requires phosphorylation by both PI3K and mTORC2 in order to be fully activated. In low cellular energy levels, liver kinase B1 tumor suppressor activates the adenosine monophosphate-activated protein kinase pathway, which negatively regulates the mTOR pathway through the tuberin (TSC1)/hamartin (TSC2) complex. Furthermore, PTEN is an additional negative regulator of $\mathrm{mTOR}$ [1-5].

The PI3K/mTOR pathway is often found dysregulated in cancer, promoting
\end{abstract}

cancer survival and growth. Mutations can occur in any component of the PI3K pathway, resulting in its dysregulation. In breast cancer, PIK3CA mutations have been commonly identified, occurring at a frequency of $27-36 \%$ [6]. The luminal A subtype of breast cancer had the highest frequency of PIK3CA mutation $(45 \%)$ and the basal subtype had the lowest (9\%). Additional identified aberrations resulting in the dysregulation of the PI3K/mTOR pathway include $A k t$ and PTEN mutations, or loss of PTEN expression $[7,8]$.

The concept of mTOR inhibition in breast cancer was firstly supported by preclinical evidence demonstrating activation of the PI3K/mTOR pathway after longterm estrogen deprivation [9,10]; estrogendeprived cells rely heavily on the PI3K signaling pathway, making this an important mechanism of acquired endocrine resistance. Combined use of antiestrogens (tamoxifen/letrozole) with rapamycin analogs demonstrated increased antitumor activity and reversal of endocrine resistance secondary to PI3K/mTOR activation in preclinical models [11,12].

\section{KEYWORDS}

- breast cancer • clinical

trials $\bullet$ inhibitors $\bullet$ mTOR

"In the metastatic setting, clinical trials using single-agent mTOR inhibitors demonstrated modest activity with a response rate ranging between 9 and 12\%." 
“...carefully designed large-scale clinical trials are needed to demonstrate the groups of patients benefiting the most from the inhibition of the $\mathrm{PI3K} / \mathrm{mTOR}$ pathway..."
In the metastatic setting, clinical trials using single-agent mTOR inhibitors demonstrated modest activity with a response rate ranging between 9 and $12 \%$ [13]. In an effort to enrich for PIK3CA mutations, a clinical trial of temsirolimus alone in hormone receptor-positive or HER2-positive metastatic breast cancer patients, failed to demonstrate higher activity. PIK3CA mutations were not associated with a higher response rate, however the primary tumors were associated with it that were analyzed, and not the recurrent or metastatic sites that may have obscured a true association [14]. A large Phase III trial, randomizing postmenopausal patients with metastatic hormone receptor-positive breast cancer between temsirolimus and letrozole or letrozole alone had to be closed prematurely due to lack of efficacy, disappointing the scientific community at the time. However, as the trial limited the use of mTOR inhibition in the first-line metastatic setting, the negative results were attributed to the lack of previous exposure to estrogen deprivation which would upregulate the PI3K/mTOR pathway and drive tumors to become dependent on it for their survival and growth [15]. The TAMRAD study randomized patients with prior exposure to an aromatase inhibitor (AI) in the metastatic setting, to tamoxifen alone versus combination of tamoxifen and everolimus. [16] The combined treatment was associated with higher clinical benefit rate ( 42 vs $61 \% ; p=0.045$ ) and time to progression (4.5 vs 8.6 months; hazard ratio [HR]: 0.54; 95\% CI: 0.36-0.81; $\mathrm{p}=0.002$ ). When patients were stratified based on primary hormone resistance, defined as relapse during adjuvant $\mathrm{AI}$ therapy or progression within 6 months of AI treatment in the metastatic setting versus secondary hormone resistance, defined as late relapse or progression on an AI in the metastatic setting more than 6 months after treatment, it was obvious that patients with secondary endocrine resistance were associated with a higher clinical benefit ratio and time to progression when compared with patients with primary hormonal resistance, further supporting the theory that upfront estrogen deprivation is required to prime tumors to be dependent on the $\mathrm{PI} 3 \mathrm{~K} / \mathrm{mTOR}$ pathway. The above results were further supported by the BOLERO-2 study randomizing patients with advanced hormone receptor-positive breast cancer, whose disease had progressed on a nonsteroidal AI, to combination examestane and everolimus or examestane alone, and demonstrated superior progression-free survival with the combination (4.1 vs 10.6 months; HR: 0.36; 95\% CI: 0.27-0.47; p < 0.001) [17].

There is also substantial preclinical evidence that the PI3K/mTOR pathway is also implicated in the development of resistance in HER2amplified disease. In a cohort of 55 breast cancer patients, activation of the PI3K pathway, assessed by the presence of oncogenic PIK3CA mutations or low PTEN expression, was associated with poor prognosis after trastuzumab therapy, and the combined analysis of PTEN and PIK3CA identified twice as many patients at increased risk for progression compared with PTEN alone [18]. Similarly in HER2 overexpressing cell lines, the combination of trastuzumab and the Akt inhibitor triciribine inhibited breast cancer cell growth and induced apoptosis, with similar results observed from xenograft models. The combination of trastuzumab and the mTOR inhibitor RAD001 also impeded breast cancer cell growth in vitro and in vivo [19]. In another study using various breast cancer cell lines innately resistant to trastuzumab or lapatinib, it was demonstrated that loss of PTEN or the presence of activating mutations in PI3K were associated with resistance to trastuzumab, which could be reversed by lapatinib through the dephosphorylation of AKT [20].

Encouraging results were obtained from a clinical study of everolimus in combination with paclitaxel and trastuzumab in trastuzumabrefractory HER2-positive metastatic breast cancer, which demonstrated clinical activity with an overall response rate of $44 \%$ and control of disease for 6 months or more in $74 \%$ of the patients [21]. Similarly, a Phase I study combining everolimus with weekly trastuzumab and vinorelbine in pretreated HER2-positive metastatic breast cancer revealed an overall response rate of $19.1 \%$, control rate of $83 \%$ and median progression-free survival of 7 months, approximately [22]. Using the dual PI3K/mTOR inhibitor, BEZ235, in combination with trastuzumab in patients with metastatic HER2-positive breast cancer bearing mutations in PIK3CA/PTEN or loss of PTEN by immunohistochemistry, a Phase I/Ib clinical study demonstrated a clinical benefit rate of $27 \%$. Interestingly, no associations of responses with PI3K pathway alterations were identified [23].

Despite the infrequency of PIK3CA mutations in triple-negative breast cancer, gene profiling analysis in this disease identified six distinct molecular subtypes, amongst which mesenchymal and mesenchymal stem cell-like subtypes, 
which were sensitive to dual PI3K/mTOR inhibitors [24]. Subsequently, a small Phase II trial with everolimus and carboplatin in patients with metastatic triple-negative breast cancer identified a clinical benefit rate of $38 \%$ [25].

\section{What is the way forward?}

It is becoming clear that carefully designed largescale clinical trials are needed to demonstrate the groups of patients benefiting the most from the inhibition of the PI3K/mTOR pathway; this is substantiated by the evidence supporting that prior estrogen deprivation is required to prime tumors to become dependent on the PI3K/mTOR pathway as they gradually evolve toward the development of endocrine resistance. Given the recent evidence supporting the extension of adjuvant endocrine treatment for up to 10 years in patients with highrisk hormone receptor-positive breast cancer and the lack of evidence of PI3K/mTOR inhibition in the adjuvant setting currently, it would be very interesting to quantify the potenttial benefit of the addition of everolimus to standard endocrine treatments in the adjuvant setting, after $2-3$ years of initial antiestrogens alone (ClinicalTrials identifier: NCT01805271) [26].

Immunohistochemical analysis or even gene profiling looking for aberrations of the pathway components in the primary tumor is not adequate to select appropriate patients with a high probability to respond to trialed treatments, as the results from previous clinical trials analyzing primary tumors are rather contradictive. Repeat biopsies are becoming essential and should be encouraged within clinical trials to identify and investigate biomarkers of resistance and treatment efficacy at the molecular level through deep sequencing techniques. We anticipate with great interest the results of clinical trials investigating the efficacy of dual PI3K/mTOR inhibitors, as the effects of the first generation mTOR inhibitors have thus far been rather modest, most likely due to 'molecular escape routes' of cancer cells via the activation of alternative survival pathways. Simultaneous inhibition of more than one pathway, in other words, PI3K/mTOR and IGFR is a concept that should be considered, as supported by a growing amount of preclinical evidence. The reversal of resistance to EGFR treatments would be of paramount significance for patients with HER2-positive breast cancer who have progressed on previous trastuzumab, and therefore the final results of the BOLERO-1/3 trials as well as other studies investigating the efficacy of the addition of mTOR inhibitors to ongoing chemotherapy and trastuzumab are also greatly anticipated.

The field of PI3K/mTOR inhibition in breast cancer remains highly active with many potentially exciting implications that can revolutionize the clinical management of patients. Relevant preclinical research and support of the ongoing clinical trials should be strongly recommended.

\section{Financial \& competing interests disclosure}

The authors have no relevant affiliations or financial involvement with any organization or entity with a financial interest in or financial conflict with the subject matter or materials discussed in the manuscript. This includes employment, consultancies, honoraria, stock ownership or options, expert testimony, grants or patents received or pending, or royalties.

No writing assistance was utilized in the production of this manuscript.

\section{References}

1 Ma XM, Blenis J. Molecular mechanisms of mTOR-mediated translational control. Nat. Rev. Mol. Cell Biol. 10, 307-318 (2009).

2 Alers S, Loffler AS, Wesselborg S, Stork B. Role of AMPK-mTOR-Ulk1/2 in the regulation of autophagy: cross talk, shortcuts, and feedbacks. Mol. Cell Biol. 32, 2-11 (2012).

3 Sarbassov DD, Guertin DA, Ali SM, Sabatini DM. Phosphorylation and regulation of Akt/PKB by the rictor-mTOR complex. Science 307, 1098-1101 (2005).

4 Shackelford DB, Shaw RJ. The LKB1-AMPK pathway: metabolism and growth control in tumour suppression. Nat. Rev. Cancer 9 , 563-575 (2009).
5 Gwinn DM, Shackelford DB, Egan DF et al. AMPK phosphorylation of raptor mediates a metabolic checkpoint. Mol Cell. 30, 214-226 (2008).

6 Banerji S, Cibulskis K, Rangel-Escareno C et al. Sequence analysis of mutations and translocations across breast cancer subtypes. Nature 486, 405-409 (2012).

7 Saal LH, Holm K, Maurer M et al. PIK3CA mutations correlate with hormone receptors, node metastasis, and ERBB2, and are mutually exclusive with PTEN loss in human breast carcinoma. Cancer Res. 65, 2554-2559 (2005).

8 Stemke-Hale K, Gonzalez-Angulo AM, Lluch A et al. An integrative genomic and proteomic analysis of PIK3CA, PTEN, and $A K T$ mutations in breast cancer. Cancer Res. 68, 6084-6091 (2008).

9 Martin LA, Farmer I, Johnston SR et al. Enhanced estrogen receptor (ER) alpha, ERBB2, and MAPK signal transduction pathways operate during the adaptation of MCF-7 cells to long-term estrogen deprivation. J. Biol. Chem. 278, 30458-30468 (2013).

10 Miller TW, Hennessy BT, Gonzalez-Angulo AM et al. Hyperactivation of phosphatidylinositol-3 kinase promotes escape from hormone dependence in estrogen receptor-positive human breast cancer. J. Clin. Invest. 120, 2406-2413 (2010).

11 Boulay A, Rudloff J, Ye J et al. Dual inhibition of mTOR and estrogen receptor signaling 
in vitro induces cell death in models of breast cancer. Clin. Cancer Res. 11, 5319-5328 (2005).

12 Clark AS, West K, Streicher S, Dennis PA. Constitutive and inducible Akt activity promotes resistance to chemotherapy, trastuzumab, or tamoxifen in breast cancer cells. Mol. Cancer Ther. 1, 707-717 (2002).

13 Ellard SL, Clemons M, Gelmon KA et al. Randomized Phase II study comparing two schedules of everolimus in patients with recurrent/metastatic breast cancer: NCIC Clinical Trials Group IND.163. J. Clin. Oncol. 27, 4536-4541 (2009).

14 Fleming GF, Ma CX, Huo D et al. Phase II trial of temsirolimus in patients with metastatic breast cancer. Breast Cancer Res. Treat. 136, 355-363 (2012).

15 Chow LWC, Sun Y, Jassem J et al. Phase 3 study of temsirolimus with letrozole or letrozole alone in postmenopausal women with locally advanced or metastatic breast cancer. Breast Cancer Res. Treat. 97(Suppl. 1), Abstract 6091 (2006).

16 Bachelot T, Bourgier C, Cropet C et al. Randomized Phase II trial of everolimus in combination with tamoxifen in patients with hormone receptor-positive, human epidermal growth factor receptor 2-negative metastatic breast cancer with prior exposure to aromatase inhibitors: a GINECO study. J. Clin. Oncol. 30, 2718-2724 (2012).

17 Baselga J, Campone M, Piccart M et al. Everolimus in postmenopausal hormonereceptor-positive advanced breast cancer. N. Engl. J. Med. 366(6), 520-529 (2012).

18 Berns K, Horlings HM, Hennessy BT et al. A functional genetic approach identifies the PI3K pathway as a major determinant of trastuzumab resistance in breast cancer. Cancer Cell. 12(4), 395-402 (2007).

19 Lu CH, Wyszomierski SL, Tseng LM et al. Preclinical testing of clinically applicable strategies for overcoming trastuzumab resistance caused by PTEN deficiency. Clin. Cancer Res. 13(19), 5883-5888 (2007).

20 O'Brien NA, Browne BC, Chow L et al. Activated phosphoinositide 3-kinase/AKT signaling confers resistance to trastuzumab but not lapatinib. Mol. Cancer Ther. 9(6), 1489-1502 (2010).

21 Andre F, Campone M, O'Regan R et al. Phase I study of everolimus plus weekly paclitaxel and trastuzumab in patients with metastatic breast cancer pretreated with trastuzumab. J. Clin. Oncol. 28, 5110-5115 (2010).

22 Jerusalem G, Fasolo A, Dieras V et al. Phase I trial of oral mTOR inhibitor everolimus in combination with trastuzumab and vinorelbine in pre-treated patients with HER2-overexpressing metastatic breast cancer. Breast Cancer Res. Treat. 125, 447-455 (2011).

23 Krop IE, Saura C, Ahnert JR et al. A Phase I/IB dose-escalation study of BEZ235 in combination with trastuzumab in patients with PI3-kinase or PTEN altered HER2+ metastatic breast cancer. J. Clin. Oncol. 30 (Suppl.), Abstract 508 (2012).

24 Lehmann BD, Bauer JA, Chen X et al. Identification of human triple-negative breast cancer subtypes and preclinical models for selection of targeted therapies. J. Clin. Invest. 121, 2750-2767 (2011).

25 Singh JC, Volm M, Novik Y. Efficacy of RAD001/carboplatin in triple-negative metastatic breast cancer: a Phase II study. J. Clin. Oncol. 30 (Suppl. 27), Abstract 108 (2012).

26 ClinicalTrials. www.clinicaltrials.gov 